\title{
Short-term outcomes of laparoscopic versus open proximal gastrectomy with double-tract reconstruction for Siewert type II and III adenocarcinoma of the esophagogastric junction: a retrospective observational study of consecutive patients
}

\author{
Fei Ma ${ }^{1 \#}$, Weifeng Wang ${ }^{2 \#}$, Dandan Guo ${ }^{3}$, Yonglei Zhang ${ }^{1}$, Liangqun Peng ${ }^{1}$, Qi Ma ${ }^{1}$, Sheqing Ji ${ }^{1}$, \\ Junhui Chai ${ }^{1}$, Yawei Hua ${ }^{1}$, Xiaobing Chen ${ }^{4}$, Hui Wang ${ }^{4}$, Shuning Xu ${ }^{4}$, Qian $\mathrm{Li}^{4}$, Suxia Luo ${ }^{4}$, \\ Hiroharu Yamashita ${ }^{5}$, Kheng Tian $\mathrm{Lim}^{6}$, Tian $\mathrm{Li}^{7}$, Bin Zhang ${ }^{1}$
}

${ }^{1}$ Department of General Surgery, The Affiliated Tumor Hospital of Zhengzhou University, Zhengzhou, China; ${ }^{2}$ Department of Gastrointestinal Surgery, Xinchang Hospital Affiliated to Wenzhou Medical University, Shaoxing, China; ${ }^{3}$ Department of Radiology, The Third Affiliated Hospital of Zhengzhou University, Zhengzhou, China; ${ }^{4}$ Department of Medical Oncology, The Affiliated Tumor Hospital of Zhengzhou University, Zhengzhou, China; ${ }^{5}$ Department of Digestive Surgery, Nihon University School of Medicine, Tokyo, Japan; ${ }^{6}$ Department of Surgery, Khoo Teck Puat Hospital, Singapore, Singapore; ${ }^{7}$ School of Basic Medicine, Fourth Military Medical University, Xi'an, China

Contributions: (I) Conception and design: F Ma, B Zhang; (II) Administrative support: Y Hua, S Luo; (III) Provision of study materials or patients: B Zhang, Y Zhang, Q Ma, S Ji, J Chai, X Chen, Q Li; (IV) Collection and assembly of data: D Guo, L Peng, F Ma; (V) Data analysis and interpretation: D Guo, L Peng, H Wang, S Xu; (VI) Manuscript writing: All authors; (VII) Final approval of manuscript: All authors.

\#These authors contributed equally to this work.

Correspondence to: Bin Zhang. Department of General Surgery, The Affiliated Tumor Hospital of Zhengzhou University, No. 127 Dongming Rd., Zhengzhou 450008, China. Email: zlyyzhangbin1055@zzu.edu.cn; Tian Li. School of Basic Medicine, Fourth Military Medical University, No. 169 Changle West Rd., Xi'an 710032, China. Email: fmmult@foxmail.com.

Background: To investigate the safety and merits of laparoscopic proximal gastrectomy with double-tract reconstruction (LPG-DT) for Siewert type II and III adenocarcinoma of the esophagogastric junction (AEG). Methods: Retrospective analysis of the clinical data of 100 consecutive patients with Siewert II and III AEG treated at the Affiliated Tumor Hospital of Zhengzhou University from October 2010 to October 2019 was performed. Out of these patients, 69 underwent open proximal gastrectomy with double-tract reconstruction (OPG-DT), while 31 underwent LPG-DT. The clinicopathological characteristics, perioperative data, and short-term outcomes of the two groups were compared. A P value $<0.05$ was considered statistically significant.

Results: Males accounted for $87 \%$ of all patients. Lymph nodes (LNs) count, time to first meal, postoperative length of stay, and postoperative complications were similar between the OPG-DT and LPGDT group. flatus time was significantly shorter in the LPG-DT group $(\mathrm{P}<0.05)$, while the duration of operation was significantly shorter in the the OPG-DT group $(\mathrm{P}<0.001)$. Furthermore, the LPG-DT group has less blood loss, shorter flatus time, and lower postoperative-day-5 white blood cell (WBC) count and C-reactive protein (CRP) levels $(\mathrm{P}<0.05)$.

Conclusions: Although LPG-DT took longer to perform, its advantages of reduced blood loss and less surgical stress reflected on inflammatory markers supports an acceptable surgical option for Siewert II and III AEG.

\footnotetext{
Keywords: Adenocarcinoma of the esophagogastric junction (AEG); proximal gastrectomy (PG); digestive tract reconstruction; double-tract reconstruction (DT)
}

Submitted Nov 06, 2020. Accepted for publication Feb 10, 2021.

doi: $10.21037 / \mathrm{atm}-21-130$

View this article at: http://dx.doi.org/10.21037/atm-21-130 


\section{Introduction}

The incidence of adenocarcinoma of the esophagogastric junction (AEG) has increased over the past few decades $(1,2)$. Surgery-based treatment is an effective treatment strategy for AEG and the main way to cure the disease (3). Despite extensive research, the scope of surgical resection and reconstruction of AEG is still controversial. Total gastrectomy (TG) and proximal gastrectomy (PG) are the common surgical methods for Siewert II and III AEG. While TG can provide an adequate resection margin and radical lymphadenectomy, it can also result in inadequate nutrition after surgery. A recent Japanese study showed no significant differences in the oncological safety between TG and PG for Siewert type II and III AEG (4). More importantly, several studies have shown that PG can improve nutritional status and quality of life compared with TG in early gastric cancer patients, likely due to preservation of the physiological function of the gastric remnant $(5,6)$. Therefore, these benefits of PG may support the use of this surgical approach in suitable cases of Siewert type II and III AEG. The role of PG has recently attracted broad interest from gastrointestinal surgical oncologists in Eastern countries, including China.

It is recognized that $\mathrm{PG}$ with esophagogastrostomy has been associated with refractory reflux esophagitis and anastomotic stricture (7), and these complications have limited its broader deployment. Other reconstruction methods have been explored to solve these complications, including side overlap esophagogastrostomy, doubleflap techniques, jejunal interposition, double-tract reconstruction (DT), and others (8). PG with DT has recently become more prevalent in clinical practice due to its ability to confer comparable clinical benefits (9). With the development of laparoscopic technology, laparoscopy is more and more widely used in radical resection of gastric cancer. Nonetheless, the evidence and safety of laparoscopic PG with DT (LPG-DT) remains unclear.

This retrospective study thus reviewed the clinical data of 100 consecutive patients from October 2010 and October 2019, and compared the short-term outcomes between LPG-DT and open PG with DT (OPG-DT) for patients with Siewert type II and III AEG.

We present the following article in accordance with the STROBE reporting checklist (available at http://dx.doi. org/10.21037/atm-21-130).

\section{Methods}

\section{Patient population}

The clinical data of consecutive patients with Siewert type II and III AEG treated by the same team of surgeons at The Affiliated Tumor Hospital of Zhengzhou University from October 2010 and June 2019 were included in the study. This was a retrospective case-control study and the requirement for informed consent was waived. All procedures performed in this study involving human participants were in accordance with the Declaration of Helsinki (as revised in 2013). The study was approved by the Ethics Committee of The Affiliated Tumor Hospital of Zhengzhou University (No. 2019156). This study didn't involve the biological tissues and specimens of patients, and informed immunity. Patients' selection was based on the following inclusion criteria: (I) Siewert type II and III AEG confirmed by gastroscopy and biopsy before surgery; (II) tumor size $<5$ cm; (III) abdominal enhanced computed tomography (CT) diagnosis of cT1-2N0M0; (IV) aged 18-80 years old; (V) no prior history of malignant tumors or presence of serious underlying disease, Eastern Cooperative Oncology Group (ECOG) physical performance score 0 or 1 , and American Society of Anesthesiologists (ASA) physical status class $\leq 2$; (VI) informed consent. Meanwhile, the exclusion criteria for patients were the following: (I) clinical stage > cT3 or $\mathrm{N}+$ (II) a history of previous radical surgery; (III) a history of neoadjuvant therapy; (IV) a history of combined organ resection; (V) presence of multiple malignant tumors; (VI) intraoperative or postoperative pathological diagnosis of upper margin involvement; (VII) incomplete follow-up data. The patient data was collected including patient's sex, age, body mass index (BMI), past medical history (hypertension, diabetes, coronary heart disease, previous surgery history), laboratory examinations, operation approach, duration of operation, intraoperative blood loss, number of lymph nodes (LNs) count, postoperative complications as defined by the Clavien-Dindo classification.

Patients were divided into two groups according to the surgical approach. Before 2014, all operations performed were laparotomies. With the advancement of laparoscopic technology, laparoscopic surgery was gradually introduced. Since 2014, the selection of laparoscopic surgery depends on the comprehensive preoperative consideration, which may include a smaller tumor size, an esophageal infiltration length $<2 \mathrm{~cm}$, and the patient's wishes. 

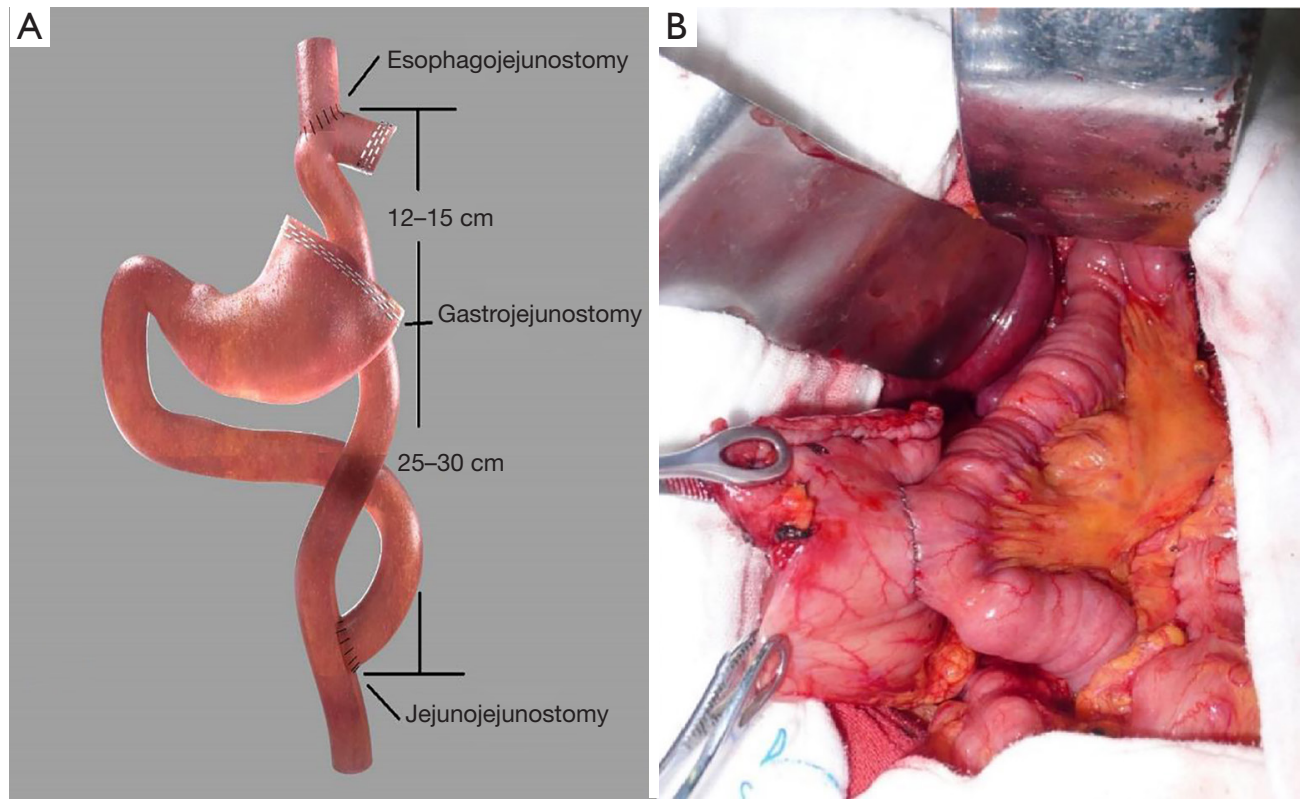

Figure 1 Schematic diagram and anastomosis in the laparotomy group. (A) Schematic diagram of PG-DT. (B) Anastomosis in the laparotomy group. PG-DT, proximal gastrectomy with double-tract reconstruction.

\section{Operative procedures}

All operations were performed by the same team of four surgeons.

\section{Extent of resection and LN dissection}

The extent of $\mathrm{LN}$ dissection was performed with extended D1 + lymphadenectomy based on the Japanese gastric cancer treatment guidelines. but according to our previous surgical experience, we included dissection of the LNs at station no. 1, 2, 3, 4sa, 4sb, 5, 7, 8a, 9, 11p, 12a, and incomplete no. $4 \mathrm{~d}$ (outside of greater curvature) and no. 6 (anterosuperior LNs along gastroepiploic vessels). The anastomosis was performed using the DT method in the following fashion: The patient was placed in a supine position under general anesthesia, and a midline incision was made in the upper abdomen. After a thorough examination to exclude abdominal metastasis, PG was performed. The gastro-colic omentum was separated to the edge of the station no. $4 \mathrm{~d}$ and no. $6 \mathrm{LNs}$, and the right gastroepiploic artery and vein were preserved. The station no. 12a LNs were dissected, the right gastric artery and vagal nerves were cut off, and the no. 5 and no. 3b LNs were dissected. The lesser omentum was then dissected to the right crus of the diaphragmatic. Endoscopic linear cutter staplers were used at the predetermined resection margin to cut off the stomach while ensuring adequate resection margin. Station no. 7, 8a, 9, and $11 \mathrm{p} \mathrm{LNs} \mathrm{were} \mathrm{then} \mathrm{dissected,} \mathrm{and} \mathrm{the} \mathrm{left} \mathrm{gastric} \mathrm{artery,}$ coronary vein, and posterior gastric vessels were ligated. Next, the left gastroepiploic vessels were ligated, and the station no. 4sb LNs were dissected. Finally, the short gastric vessels were ligated, and the station no. 10 and 4sa LNs were dissected and expanded to the left crus of the diaphragmatic.

\section{Reconstruction methods}

Patients were grouped according to either laparotomy or laparoscopic anastomotic reconstruction methods.

In OPG-DT group, the anvil of the circular stapler was separately placed into the stump of the esophagus using a purse-string suturing device and the gastric remnant, the lower end of the esophagus was cut off with complete PG. The jejunum was divided about $25 \mathrm{~cm}$ below the ligament of Treitz, and a circular stapler was inserted into the distal jejunal opening. Gastrojejunostomy was performed using circular stapler on the posterior wall of the gastric remnant. About $15 \mathrm{~cm}$ above gastrojejunal anastomosis, the esophagojejunostomy was performed using circular stapler, after which the linear stapler was used to close the jejunal opening (Figure 1). Hand-sewn jejunojejunostomy was performed about $45 \mathrm{~cm}$ below the esophagojejunostomy. All anastomoses were reinforced with second layer of either continuous or interrupted sutures. A silicone drainage tube 

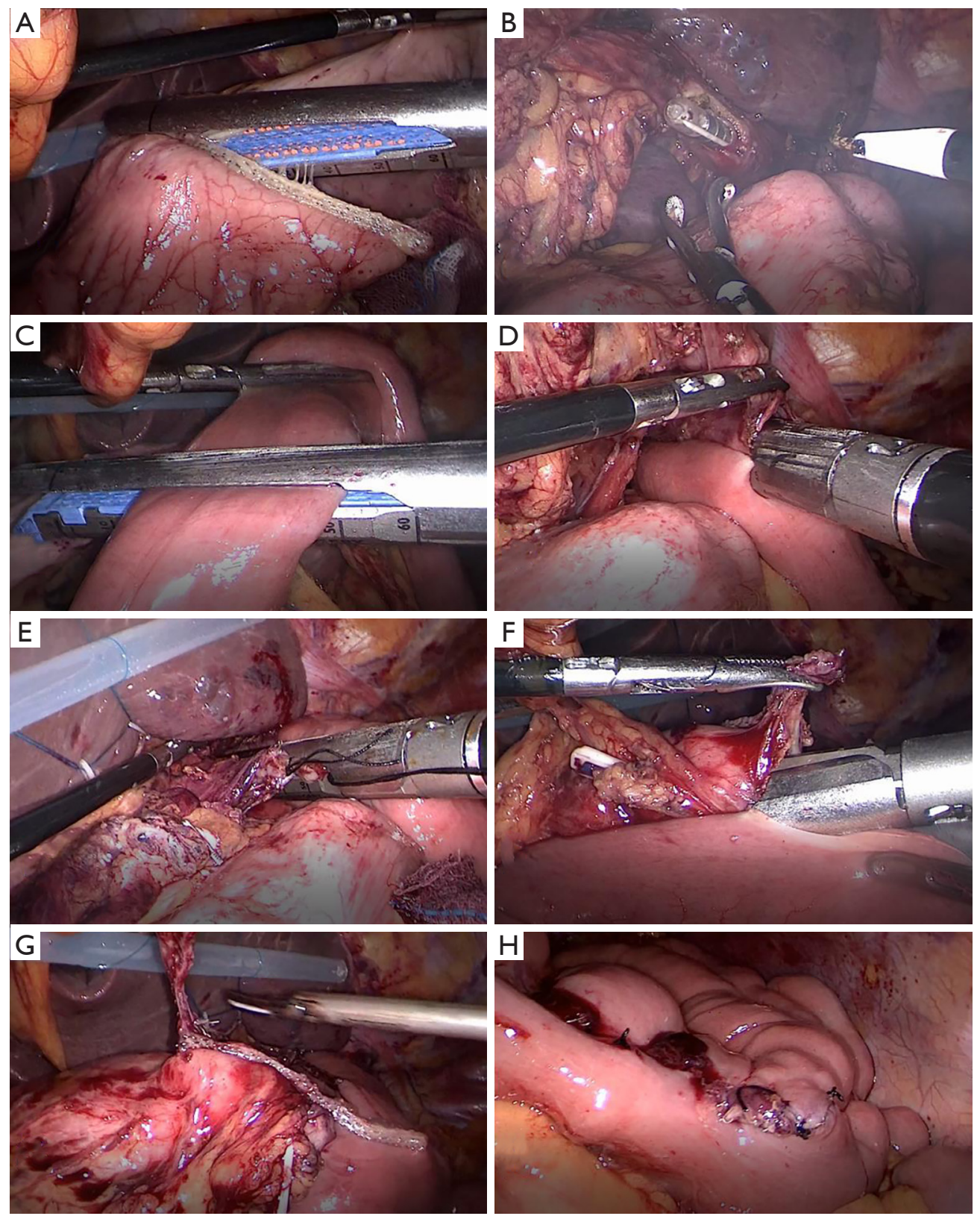

Figure 2 Laparoscopic surgery procedure. (A) Stomach cut off by laparoscopic linear staplers. (B) Guidance of gastric tube. (C) Jejunum cut off at about $25 \mathrm{~cm}$ below the Treitz ligament. (D) Over-lap esophagojejunostomy. (E) Common opening closed by laparoscopic linear staplers. (F) Side-to-side gastrojejunostomy. (G) Common opening closed by laparoscopic linear staplers. (H) Hand-sewn jejunojejunostomy.

was inserted at the posterior parts of esophagojejunostomy and led out from the right abdominal wall behind the hepatoduodenal ligament.

In LPG-DT group, each intended anastomosis site was marked on the alimentary limb in similar fashion to the conventional open approach in which esophagojejunostomy and gastrojejunostomy were performed using a laparoscopic linear stapler. Jejunojejunostomy was performed using hand-sewn anastomosis extracorporeally via minilaparotomy (Figure 2).

\section{Outcomes}

The primary outcomes were perioperative complications, 
while the secondary outcomes were surgical stress indicators and reflux symptoms.

\section{Postoperative therapy and follow-up}

The visual analogue scale (VAS) was used to evaluate the degree of pain in the following manner: (I) resting state score: evaluation of the patient pain level when lying supine; (II) activity status score: evaluation of patient's pain intensity during coughing according to the breathing training method taught to the patient.

Pain treatment consisted primarily of multimodal analgesia including preoperative ultrasound-guided ropivacaine abdominal transverse fascia nerve block, postoperative patientcontrolled electronic intravenous analgesia pump, analgesic application of sufentanil at a concentration of $0.02 \mu \mathrm{g} / \mathrm{kg} / \mathrm{mL}$, $2 \mathrm{~mL}$ analgesic entered the vein when analgesia pump was pressed. Meanwhile, $40 \mathrm{mg}$ of parecoxib was infused intravenously every 12 hours. We set the analgesic pump to have only 1 effective compression every 15 minutes.

All patients were followed up at the outpatient clinics every 3 to 6 months. Physical examination and hematologic analysis were performed during each visit, including tumor marker carcinoembryonic antigen (CEA), carbohydrate antigen 72-4 (CA72-4), and carbohydrate antigen 19-9 (CA19-9). Chest and abdominal CT scan was performed every 6 months or when recurrence was suspected. Endoscopy was performed every 12 months, and these endoscopic findings were graded using the Los Angeles classification of reflux esophagitis (10).

\section{Statistical analyses}

The continuous variables were all normally distributed and are therefore expressed as mean and standard deviations, while categorical variables are expressed as $\mathrm{n}(\%)$. The OPG-DT and LPG-DT groups were compared using Student's $t$-test for continuous variables and chi-squared test or Fisher's exact test for categorical variables. The MannWhitney U nonparametric test was used for grade count data between the groups. All analyses were conducted using SPSS for Windows version 21.0 (SPSS/IBM, Chicago, IL, USA). P values $<0.05$ were considered to indicate statistically significant differences.

\section{Results}

A total of 100 patients underwent radical PG-DT, consisted of 50 patients in each of Siewert type II and III AEG. No tumor-related death occurred in all patients 1 year after operation. There were 69 patients who underwent OPGDT, and 31 patients who underwent LPG-DT group. The clinicopathological characteristics of the patients are shown in Table 1.

The longer operative duration in the LPG-DT group was statistically significant than in the OPG-DT group $(\mathrm{P}<0.001)$. With increasing operative experience over time, the operation duration improved and plateau out during this period. The amount of intraoperative blood loss in the LPG-DT group was significantly less than in the OPGDT group and the flatus time in LPG-DT group was significantly shorter than in the OPG-DT group. There was no statistical difference between the two groups in terms of the number of LNs count, time to first meal, discharge time, or complications (11) as shown in Table 2.

In terms of the assessment of postoperative pain, a total of 71 patients were eligible for analysis due to the use of multimodal analgesia beginning January 2004. After the anesthesia nurses provided preoperative analgesia education to the patients, and the treatment was applied, the Assessment of pain as shown in Table 3 and the application of intravenous pain pump as shown in Table 4. The resting state VAS scores were found to not be statistically different between the two groups at $24(\mathrm{P}=0.365), 48(\mathrm{P}=0.159)$, and $72(\mathrm{P}=0.116)$ hours. However, the activity status VAS score in the LPG-DT group was significantly lower than in the OPG-DT group at $24(\mathrm{P}=0.004), 48(\mathrm{P}=0.002)$, and 72 $(\mathrm{P}<0.001)$ hours, and there were also statistical differences in the application time of the analgesic pump and the number of additional compressions. In the open laparotomy group, the active VAS score was higher, the application time of the analgesic pump was longer, and the number of additional compressions was greater.

The average WBC levels within 7 days before surgery (pre-op) in the LPG-DT group and OPG-DT group were not significantly different $\left(6.33\right.$ vs. $6.19 \times 10^{9} / \mathrm{L}$, $\mathrm{P}>0.05)$, but they were significantly different 1 -day $(9.17 \mathrm{vs}$. $\left.11.68 \times 10^{9} / \mathrm{L}, \mathrm{P}<0.05\right), 3$-day $\left(7.44\right.$ vs. $\left.9.29 \times 10^{9} / \mathrm{L}, \mathrm{P}<0.05\right)$, and 5 -day after surgery $\left(6.86\right.$ vs. $\left.8.59 \times 10^{9} / \mathrm{L}, \mathrm{P}<0.05\right)$ as shown in Figure $3 \mathrm{~A}$. The average $\mathrm{C}$-reactive protein (CRP) levels within 7-day before surgery in the LPGDT group and OPG-DT group were not significantly different (3.93 vs. $4.46 \mathrm{mg} / \mathrm{L}, \mathrm{P}>0.05$ ), but they were significantly different in the LPG-DT group and OPGDT group 1-day after surgery (86.46 vs. $112.34 \mathrm{mg} / \mathrm{L}$, $\mathrm{P}<0.05)$, 3-day (71.57 vs. $94.55 \mathrm{mg} / \mathrm{L}, \mathrm{P}<0.05)$, and 5 -day 
Page 6 of 12

Ma et al. Safety and merits of LPG-DT for Siewert type II and III AEG

Table 1 The clinicopathological characteristics of the patients

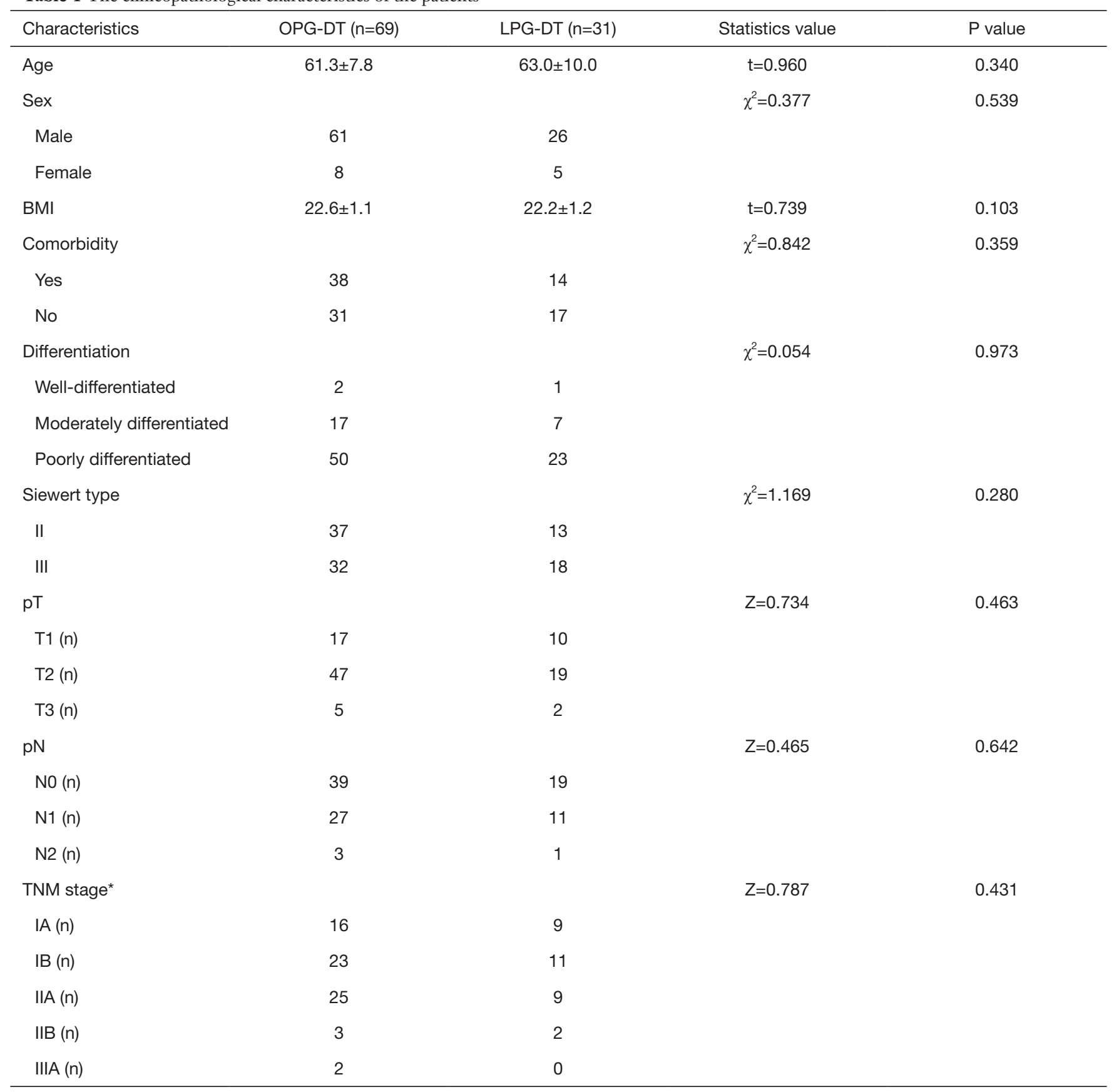

* means TNM staging according to the UICC TNM staging 8th edition. OPG-DT, open proximal gastrectomy with double-tract reconstruction; LPG-DT, laparoscopic proximal gastrectomy with double-tract reconstruction; BMI, body weight index; TNM, tumor, nodes, metastasis; UICC, union for international cancer control. 
Table 2 Operation and recovery in perioperation

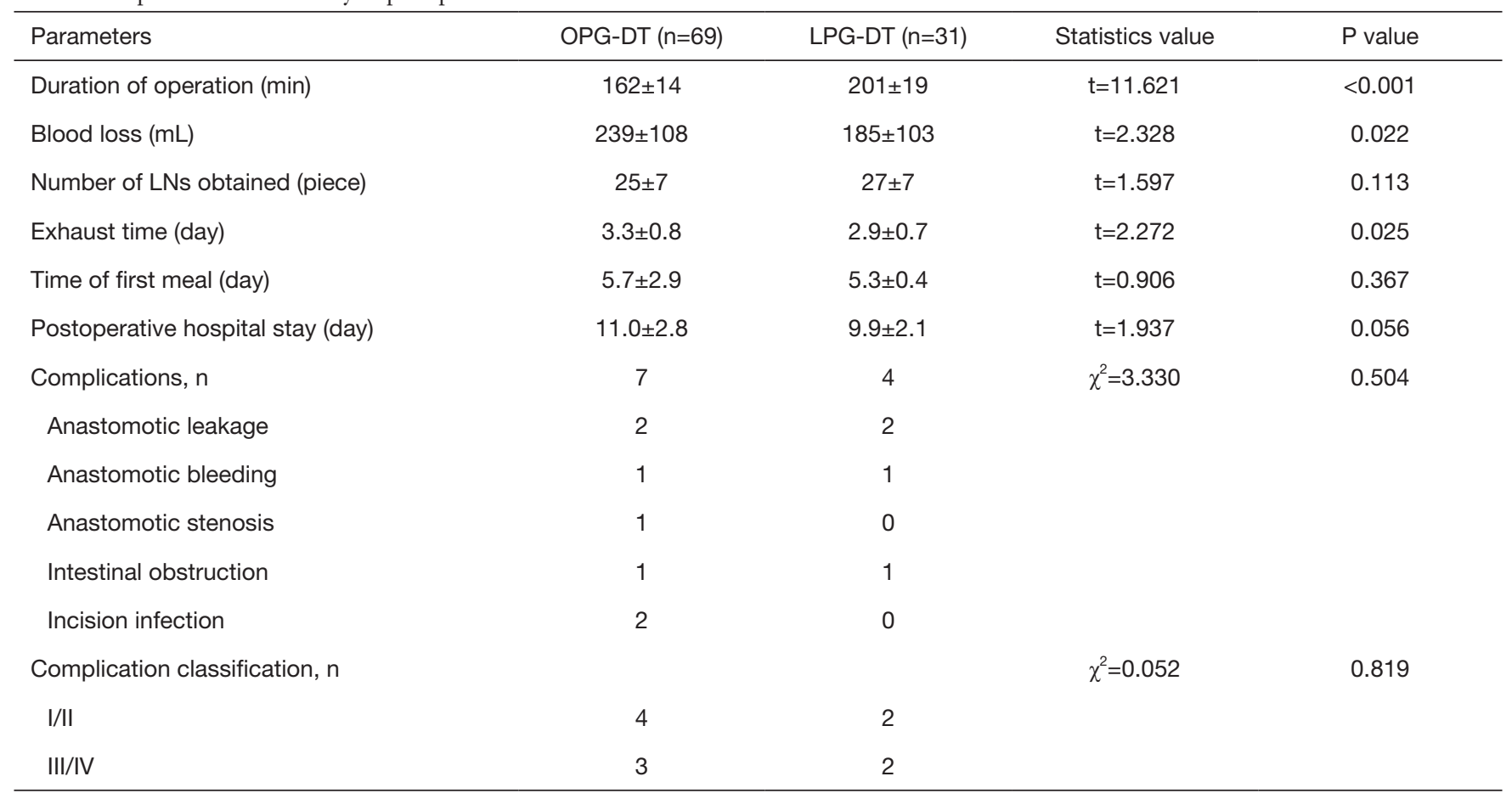

OPG-DT, open proximal gastrectomy with double-tract reconstruction; LPG-DT, laparoscopic proximal gastrectomy with double-tract reconstruction; LN, lymph node.

Table 3 Assessment of pain

\begin{tabular}{|c|c|c|c|c|c|c|c|c|}
\hline Postoperative time (h) & \multicolumn{4}{|c|}{ Resting VAS } & \multicolumn{4}{|c|}{ Activity VAS } \\
\hline 24 & $3.0 \pm 1.1$ & $2.8 \pm 1.1$ & $t=0.912$ & 0.365 & $5.3 \pm 1.5$ & $4.2 \pm 1.4$ & $t=2.985$ & 0.004 \\
\hline 48 & $2.9 \pm 1.1$ & $2.6 \pm 0.9$ & $t=1.425$ & 0.159 & $5.0 \pm 1.4$ & $4.0 \pm 1.3$ & $t=3.139$ & 0.002 \\
\hline 72 & $2.5 \pm 0.6$ & $2.2 \pm 0.7$ & $t=1.593$ & 0.116 & $4.7 \pm 1.6$ & $3.4 \pm 1.2$ & $\mathrm{t}=3.773$ & $<0.001$ \\
\hline
\end{tabular}

VAS, visual analogue scale; OPG-DT, open proximal gastrectomy with double-tract reconstruction; LPG-DT, laparoscopic proximal gastrectomy with double-tract reconstruction.

Table 4 Application of intravenous pain pump

\begin{tabular}{|c|c|c|c|c|}
\hline Intravenous pain pump & OPG-DT $(n=45)$ & LPG-DT $(n=26)$ & Statistics value & $P$ value \\
\hline Extra presses & $5.0 \pm 1.4$ & $3.6 \pm 1.2$ & $\mathrm{t}=4.140$ & $<0.001$ \\
\hline
\end{tabular}

OPG-DT, open proximal gastrectomy with double-tract reconstruction; LPG-DT, laparoscopic proximal gastrectomy with double-tract reconstruction. 

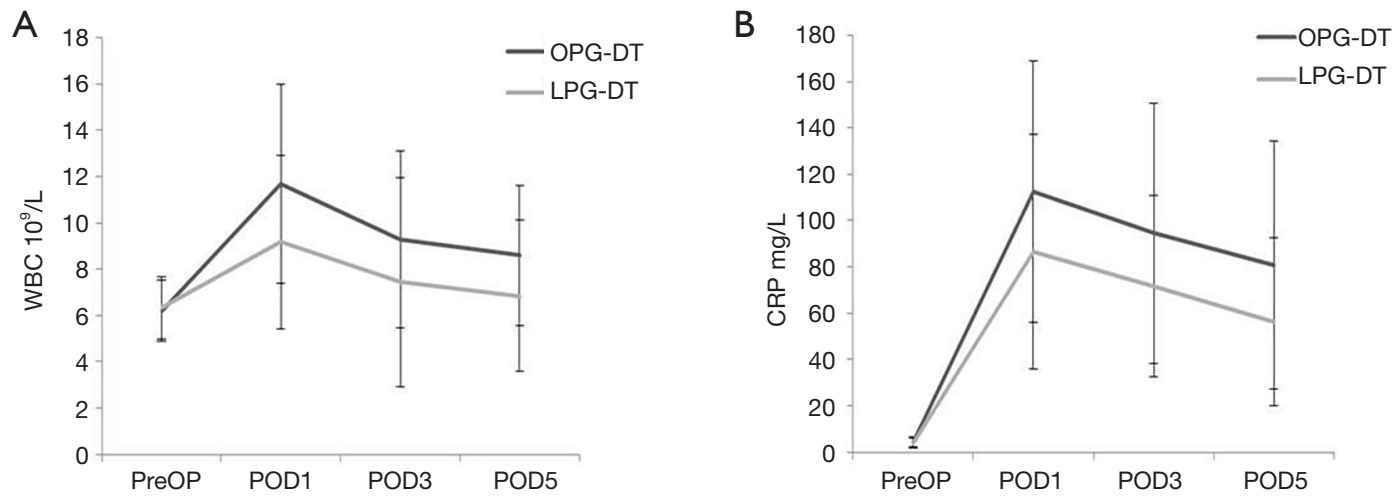

Figure 3 Changes of stress index. OPG-DT, open proximal gastrectomy with double-tract reconstruction; LPG-DT, laparoscopic proximal gastrectomy with double-tract reconstruction; WBC, white blood cell; CRP, C-reactive protein; POD, postoperative day.

Table 5 Assessment of reflux esophagitis

\begin{tabular}{|c|c|c|c|c|}
\hline Method & OPG-DT & LPG-DT & Statistics value & $P$ value \\
\hline 1 & 63 & 27 & & \\
\hline ॥ & 1 & 1 & & \\
\hline III & 0 & 0 & & \\
\hline Los Angeles classification of reflux esophagitis & & & $Z=0.394$ & 0.693 \\
\hline A & 4 & 2 & & \\
\hline B & 1 & 1 & & \\
\hline $\mathrm{C}$ & 0 & 0 & & \\
\hline
\end{tabular}

OPG-DT, open proximal gastrectomy with double-tract reconstruction; LPG-DT, laparoscopic proximal gastrectomy with double-tract reconstruction.

(56.13 vs. $80.83 \mathrm{mg} / \mathrm{L}, \mathrm{P}<0.05)$ as shown in Figure $3 B$.

Furthermore, 64 patients $(92.75 \%)$ in the OPG-DT group and 28 patients $(90.32 \%)$ in the LPG-DT group underwent gastroscopy 1 year after surgery. Among them, there was 1 patient with reflux symptoms in the OPG-DT group and none in the LPG-DT group. However, according to endoscopic LA classification of reflux esophagitis, there were 5 patients with esophagitis (4 LA grade A and $1 \mathrm{LA}$ grade B) in the OPG-DT group, versus 3 patients (2 LA grade A and 1 LA grade B) in the LPG-DT group as shown in Table 5 which includes Visick score.

\section{Discussion}

\section{Main findings}

The present study was performed to clarify the safety and merits of LPG-DT for Siewert type II and III AEG patients with clinical stages T1-2N0M0. with an explicit focus on surgical outcomes and perioperative therapy. There was no difference between LPG-DT and OPGDT in perioperative complications and reflux esophagitis. More importantly, those patients who underwent LPG-DT had favorable outcomes with less intraoperative blood loss, 
shorter postoperative gastrointestinal function recovery time, less postoperative pain and less surgical stress.

\section{Interpretation}

The main challenges in performing PG-DT are reflected in the duration of the operations and the total blood loss. In LPG-DT, the technical complexity of performing three laparoscopic anastomoses were increased and these pose challenges to the operating surgeons. However, as we increased our operative experience, these technical difficulties became relatively manageable. For instance, at the beginning of our LPG-DT, the operation time was 470 minutes. After optimizing the operative steps, the operation time was reduced to an average of 200 minutes. This improvement in operative duration is similar to that described by Uyama et al. (12). In our experience, firstly we discovered that cutting the crus of diaphragm can allow better exposure of the esophagogastric junction around the hiatus especially in obese patients. Intra-operative endoscopy also helped to determine the safe level of transhiatal esophageal transection. Secondly, we found that gentle pressure from the nasogastric tube towards the esophageal stapled stump allowed better traction, visualization and less damage to the esophageal stump. The nasogastric tube also allowed guidance of safe stapling blade entry into the esophageal lumen and effectively avoiding entering any false cavity between the esophageal muscle layers. In some patients with smaller jejunal lumen, we used continuous barbed suture for the anastomoses. Although the operating duration of LPG-DT was acceptably longer than OPG-DT, the former has less bleeding. These findings support the advantages of using laparoscopic surgery as confirmed by other studies (13). The type and postoperative complications according to Clavien-Dindo classification were acceptable and similar between the two groups, which was consistent with the findings reported by Makuuchi et al. (14). There was a low incidence of anastomotic-related complications which maybe attributed to second layer of suture reinforcement on the anastomosis. The single case of delayed anastomotic stenosis in the OPG-DT group was caused by an earlier anastomotic leak. There was a case of intestinal obstruction in the LPG-DT group related to adhesions. At 1 year postoperative follow up, we performed upper gastrointestinal endoscopy to evaluate the refluxrelated esophagitis and found no difference between the two groups according to Visick score and Los Angeles classification of reflux esophagitis.
The recommendations on the extent of lymphadenectomy for proximal gastric cancer and AEG remain controversial $(15,16)$. Nonetheless, the risk of $\mathrm{LN}$ metastases ranges notably according to pathological characteristics. In early gastric cancer unsuitable for endoscopic resection, the Japanese Gastric Cancer Association (JGCA) guidelines recommend a D1 or D1 plus lymphadenectomy when LNs are clinically negative and D2 lymphadenectomy when LNs are clinically positive. It is essential to note that the definitions of the D2 level of LNs dissection are only for TG and distal gastrectomy and those for PG have not been included. Locally advanced gastric cancer has a high tendency to spread to the surrounding LNs and the extend of LNs dissection has been debated for many years between surgeons from the Eastern and Western countries (17). D2 lymphadenectomy has been considered the standard procedure in Japan since the 1960's. TG maybe seen as an excessive treatment in some of the relatively early proximal gastric cancer cases due to its effect on the quality of life. Prior to 2010, there was little consensus or guidance from the clinical research literature concerning the scope of surgical LNs dissection for locally advanced proximal gastric cancer or Siewert type II and III AEG, and most patients ended up having TG and D2 lymphadenectomy.

We also found that there was a discrepancy between preoperative staging scans and postoperative pathology that arose for several clear reasons. Such as the degree of gastric distention during CT scan, scan thickness of CT, the lack of ultrasonic gastroscope at that time and subjective judgment of doctors in imaging diagnosis. What's more, perhaps clinical under-staging of nodal disease from conventional CT scan, because some LNs $<1 \mathrm{~cm}$ harbor malignant cells as confirmed by histology. Therefore, by referring to the Japanese Gastric Cancer Guidelines at 1998 (18), we developed our extent of LNs dissection for PG, which was similar to the D2 lymphadenectomy for TG, including dissection of the LNs at stations no. 1, 2, 3, 4sa, 4sb, 5, 7, 8a, 9, 11p, and 12a, but incomplete station no. $4 \mathrm{~d}$ (outside of greater curvature) and station no. 6 (anterosuperior LNs along right gastroepiploic vessels), which might be regarded as incomplete D2 lymphadenectomy for PG. We observed that such incomplete D2 lymphadenectomy did not increase the patient's surgical and oncological risk. In addition, our previous study found that PG-DT could improve the nutritional status and quality of life of patients $(19,20)$. In a nation-wide retrospective study for esophagogastric junction carcinoma in stomach-predominant cancer in Japan, 
Yamashita et al. (21) reported the incidences of metastatic LNs at station no. 4sa, 4sb, 4d, 5, and 6 were lower than $1 \%$ even in patients with high LNs dissection rates. Results from the China National Cancer Center Gastric Cancer Database from 1997 to 2017 revealed that proximal gastric cancer patients were more likely to be in later pT stage and have LNs metastasis (22). Wang et al. (23) reported the rates of metastasis in the LNs stations in advanced proximal gastric cancer ranged from high to low in the LNs station no. $1,2,3,4,7,8 \mathrm{a}, 10,11,6$, and 5 groups. For patients at pT1-3, the positive rate for LNs station no. 5 and 6 was $15.1 \%$. Based on this evidence of postoperative pathological staging, we have adopted this extend of LNs dissection.

Surgical trauma and stress-related surgery—can affect the short-term outcome of the patient. In our study, we found that the WBC count and CRP level in the LPG-DT group were significantly lower than those of the OPG-DT group, suggesting laparoscopic surgery can significantly reduce patient's inflammatory and stress response compared to open surgery. These findings provide a theoretical basis for enhanced recovery after surgery in LPG-DT group. Takayama et al. (24) reported similar findings and this could be a reason to explain for the earlier flatus time in the LPGDT group. Another possible explanation may be due to early ambulation of patients within 24 hours after surgery when the situation was permitted. Although there was no difference in the early ambulation time between the two groups, early activities still play a role in the recovery of intestinal function. Multimodal analgesia protocol played an important role in patients' early activities and perioperative recovery. Although our study did not show any statistically significant difference of VAS in the resting status between LPG-DT and OPG-DT after the use of multimodal analgesia, the activity status of VAS was however, significantly lower in the LPG-DT group in addition to a shorter duration and less frequent use of analgesia pump in the LPG-DT group, similar to the findings reported by Li et al. (25). This evidence indicates laparoscopic gastric surgery has an advantage in early postoperative activity when compared to open surgery.

\section{Study strengths}

To our knowledge, there are only a few published articles focusing on the safety and merits of laparoscopic LPGDT for Siewert type II and III AEG patients. The data analysis of results of OPG-DT and LPG-DT performed at our center in the last 10 years provides the evidence in the safety and anti-reflux effect of these procedures.

\section{Limitations}

There were several limitations to this study. Firstly, this study was retrospective in design and we could not avoid a certain degree of selection bias. The surgical procedure in each case was selected based on a holistic decision dependent on the surgeon, the patient, and the location of the tumor. Secondly, the data collected over a period of 10 years was of modest sample size derived from a single institution. The improvement in surgical experience and new technological surgical devices may have contributed to safer and shorter duration of operations. Thirdly, the long term oncological outcomes remain to be determined due to short postoperative follow up data. Hence, the use of LPGDT for Siewert type II and III AEG must be interpreted and selected carefully by the operating surgeons.

\section{Conclusions}

Although LPG-DT takes longer to perform, this procedure has lower risk of blood loss and inflammatory response due to surgical stress when compared to OPG-DT. Therefore, LPG-DT may be an attractive choice to consider in the surgical management of Siewert type II and III AEG.

\section{Acknowledgments}

The authors appreciate the academic support from AME Gastrointestinal Oncology Collaborative Group.

Funding: The study was supported by Tackle Key Problems in Science and Technology of Henan Province (No. 192102310311), Tackle Key Problems in Medicine of Henan Province (LHGJ20190629), and the National Cancer Climbing Fund (No. NCC201816B048).

\section{Footnote}

Reporting Checklist: The authors have completed the STROBE reporting checklist. Available at http://dx.doi. org/10.21037/atm-21-130

Data Sharing Statement: Available at http://dx.doi. org/10.21037/atm-21-130

Conflicts of Interest: All authors have completed the ICMJE uniform disclosure form (available at http://dx.doi. 
org/10.21037/atm-21-130). The authors have no conflicts of interest to declare.

Ethical Statement: The authors are accountable for all aspects of the work in ensuring that questions related to the accuracy or integrity of any part of the work are appropriately investigated and resolved. This was a retrospective case-control study and the requirement for informed consent was waived. All procedures performed in this study involving human participants were in accordance with the Declaration of Helsinki (as revised in 2013). The study was approved by the Ethics Committee of The Affiliated Tumor Hospital of Zhengzhou University (No. 2019156).

Open Access Statement: This is an Open Access article distributed in accordance with the Creative Commons Attribution-NonCommercial-NoDerivs 4.0 International License (CC BY-NC-ND 4.0), which permits the noncommercial replication and distribution of the article with the strict proviso that no changes or edits are made and the original work is properly cited (including links to both the formal publication through the relevant DOI and the license). See: https://creativecommons.org/licenses/by-nc-nd/4.0/.

\section{References}

1. Bray F, Ferlay J, Soerjomataram I, et al. Global cancer statistics 2018: GLOBOCAN estimates of incidence and mortality worldwide for 36 cancers in 185 countries. CA Cancer J Clin 2018;68:394-424.

2. Zhang SW, Yang ZX, Zheng RS, et al. Incidence and mortality of stomach cancer in China, 2013. Zhonghua Zhong Liu Za Zhi 2017;39:547-52.

3. Greally M, Agarwal R, Ilson DH. Optimal management of gastroesophageal junction cancer. Cancer 2019;125:19902001.

4. Yura M, Yoshikawa T, Otsuki S, et al. Oncological safety of proximal gastrectomy for T2/T3 proximal gastric cancer. Gastric Cancer 2019;22:1029-35.

5. Chen YC, Lu L, Fan KH, et al. Proximal gastrectomy versus total gastrectomy for adenocarcinoma of the esophagogastric junction: a meta-analysis. J Comp Eff Res 2019;8:753-66.

6. Park JY, Park KB, Kwon OK, et al. Comparison of laparoscopic proximal gastrectomy with double-tract reconstruction and laparoscopic total gastrectomy in terms of nutritional status or quality of life in early gastric cancer patients. Eur J Surg Oncol 2018;44:1963-70.

7. $\mathrm{Xu} \mathrm{Y,} \mathrm{Tan} \mathrm{Y,} \mathrm{Wang} \mathrm{Y,} \mathrm{et} \mathrm{al.} \mathrm{Proximal} \mathrm{versus} \mathrm{total}$ gastrectomy for proximal early gastric cancer: a systematic review and meta-analysis. Medicine (Baltimore) 2019;98:e15663.

8. Japanese Gastric Cancer Association. Japanese gastric cancer treatment guidelines 2010 (ver. 3). Gastric Cancer 2011;14:113-23.

9. Li S, Gu L, Shen Z, et al. A meta-analysis of comparison of proximal gastrectomy with double-tract reconstruction and total gastrectomy for proximal early gastric cancer. BMC Surg 2019;19:117.

10. Lundell LR, Dent J, Bennett JR, et al. Endoscopic assessment of oesophagitis: clinical and functional correlates and further validation of the Los Angeles classification. Gut 1999;45:172-80.

11. Dindo D, Demartines N, Clavien PA. Classification of surgical complications: a new proposal with evaluation in a cohort of 6336 patients and results of a survey. Ann Surg 2004;240:205-13.

12. Uyama I, Sugioka A, Fujita J, et al. Completely laparoscopic proximal gastrectomy with jejunal interposition and lymphadenectomy. J Am Coll Surg 2000;191:114-9.

13. Best LM, Mughal M, Gurusamy KS. Laparoscopic versus open gastrectomy for gastric cancer. Cochrane Database Syst Rev 2016;3:CD011389.

14. Makuuchi R, Irino T, Tanizawa Y, et al. Esophagojejunal anastomotic leakage following gastrectomy for gastric cancer. Surg Today 2019;49:187-96.

15. Japanese Gastric Cancer Association. Japanese gastric cancer treatment guidelines 2018 (5th edition). Gastric Cancer 2021;24:1-21.

16. Inomata $M$, Shiroshita $H$, Uchida $H$, et al. Current status of endoscopic surgery in Japan: the 14th National Survey of Endoscopic Surgery by the Japan Society for Endoscopic Surgery. Asian J Endosc Surg 2020;13:7-18.

17. Cuschieri A, Fayers P, Fielding J, et al. Postoperative morbidity and mortality after D1 and D2 resections for gastric cancer: preliminary results of the MRC randomised controlled surgical trial. The Surgical Cooperative Group. Lancet 1996;347:995-9.

18. Japanese Gastric Cancer Association. Japanese Classification of Gastric Carcinoma - 2nd English Edition -. Gastric Cancer 1998;1:10-24.

19. Zhang ZD, Ma F, Zhang YL, et al. Comparison study of three methods of digestive tract reconstruction after radical gastrectomy in gastric cancer patients. Zhonghua 
Wei Chang Wai Ke Za Zhi 2013;16:1073-7.

20. Zhang B, Ji SQ, Hua YW, et al. Application of jejunal interposition after radical proximal gastrectomy, Zhonghua Zhong Liu Za Zhi 2013,35:530-3.

21. Yamashita H, Seto Y, Sano T, et al. Results of a nationwide retrospective study of lymphadenectomy for esophagogastric junction carcinoma. Gastric Cancer 2017;20:69-83.

22. Zhao L, Huang H, Zhao D, et al. Clinicopathological characteristics and prognosis of proximal and distal gastric cancer during 1997-2017 in China National Cancer Center. J Oncol 2019;2019:9784039.

23. Wang J, Wu P, Wang Z, et al. Metastatic patterns and

Cite this article as: Ma F, Wang W, Guo D, Zhang Y, Peng L, Ma Q, Ji S, Chai J, Hua Y, Chen X, Wang H, Xu S, Li Q, Luo S, Yamashita H, Lim KT, Li T, Zhang B. Short-term outcomes of laparoscopic versus open proximal gastrectomy with doubletract reconstruction for Siewert type II and III adenocarcinoma of the esophagogastric junction: a retrospective observational study of consecutive patients. Ann Transl Med 2021;9(4):352. doi: 10.21037/atm-21-130 surgical methods for lymph nodes No. 5 and No. 6 in proximal gastric cancer. Chin J Cancer Res 2019;31:171-7.

24. Takayama Y, Kaneoka Y, Maeda A, et al. Comparison of outcomes of laparoscopy-assisted and open proximal gastrectomy with jejunal interposition for early gastric cancer in the upper third of the stomach: a retrospective observational study. Asian J Endosc Surg 2018;11:329-36.

25. Li Z, Shan FAO, Ying X, et al. Laparoscopic or open distal gastrectomy after neoadjuvant chemotherapy for advanced gastric cancer: study protocol for a randomised phase II trial. BMJ Open 2018;8:e021633.

(English Language Editor: J. Gray) 